\title{
Encephalopathy caused by \\ thiamine deficiency
}

To the Editor: Antel et al..$^{[1]}$ have drawn attention to the acute encephalopathy caused by thiamine deficiency in non-alcohol-related settings. I would like to highlight a different clinical presentation that ought to be readily recognised as a possible manifestation of this deficiency, namely rapidly progressive leg weakness, by briefly describing a woman currently under our care at the Neurology Division, Groote Schuur Hospital, Cape Town, South Africa.

A 24-year-old woman pregnant with twins presented at 20 weeks' gestation with a history of 3 weeks of numbness and weakness of the legs and 1 week of tingling and numbness of the fingers. She described daily vomiting from early in her pregnancy (7 weeks), persisting for 4 months; she had been admitted briefly to the district hospital with hyperemesis gravidarum for intravenous hydration. She did not receive any vitamin supplementation. She had been too weak to walk for 3 weeks on admission, and described symptoms of paroxysmal nocturnal dyspnoea and orthopnoea. On examination, she had a sinus tachycardia with normal blood pressure and chest and cardiac auscultations. She was fully conscious, with reduced tone in the legs, absent leg reflexes, marked proximal and distal leg weakness with mild weakness of hand grip, and glove-and-stocking sensory loss involving all sensory modalities.

Laboratory investigations showed mild normochromic anaemia. Vitamin $B_{12}$ and creatine kinase levels and the results of renal and hepatic function tests were normal. Cerebrospinal fluid parameters and a chest radiograph were normal. The findings on nerve conduction studies were in keeping with a sensorimotor axonopathy.

The patient was diagnosed with thiamine deficiency neuropathy (dry beriberi) and intravenous thiamine replacement was commenced (500 mg 8-hourly for 5 days), continuing with $100 \mathrm{mg}$ twice daily orally thereafter. She improved rapidly, developing neuropathic pain requiring symptomatic treatment, and was able to walk again after 6 days. A thiamine assay taken before treatment confirmed low levels.

This case highlights the importance of recognising individuals at high risk of developing complications of thiamine deficiency, such as pregnant women with hyperemesis gravidarum. It also draws attention to that fact that the neuropathy of thiamine deficiency may occur without encephalopathy, and can mimic Guillain-Barré syndrome. ${ }^{[2]}$ Given that this is not an infrequent scenario in our hospital, we would like to raise awareness of this easily preventable, life-threatening illness, particularly among healthcare workers in antenatal clinics, and remind them of the importance of adequate thiamine replacement in patient groups at risk.

\section{Kathleen Bateman}

Neurology Division, Department of Medicine, Groote Schuur Hospital and

Faculty of Health Sciences, University of Cape Town, South Africa

kathleen.bateman@uct.ac.za

\footnotetext{
1. Antel K, Singh N, Chisholm B, Heckmann JM. Encephalopathy after persistent vomiting: Three case of non-alcohol related Wernicke's encephalopathy. S Afr Med J 2015;105(6):442-443. [http://dx.doi. org/10.7196/SAMJ.9299

2. Koike H, Iijima M, Sugiura M, et al. Alcoholic neuropathy is clinicopathologically distinct from thiamine-deficiency neuropathy. Ann Neurol 2003;54(1):19-29. [http://dx.doi.org/ 10.1002/ana.10550
} 